\title{
Patient with typical carcinoid initially diagnosed as high-grade neuroendocrine carcinoma
}

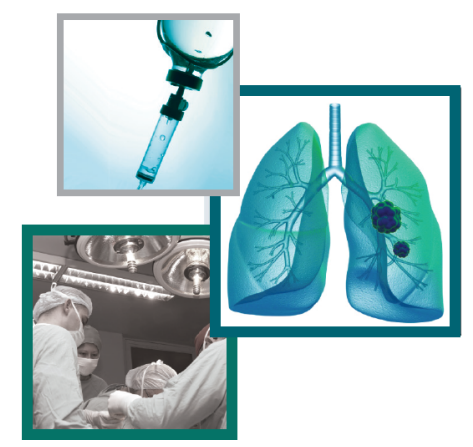

\author{
Sean Warsch ${ }^{*}, 1$ \& Mohammad Jahanzeb**,2 \\ ${ }^{1}$ University of Miami, Jackson Memorial Hospital, 1611 NW 12th Avenue, Miami, FL 33136, USA \\ ${ }^{2}$ Sylvester Comprehensive Cancer Center, University of Miami Miller School of Medicine, 1192 East Newport Center Drive, Suite \\ 100, Deerfield Beach, FL 33442-7753, USA \\ * Author for correspondence: swarsch@med.miami.edu \\ ** Author for correspondence: mjahanzeb@med.miami.edu
}

\section{Practice points}

- Diagnosis and classification of neuroendocrine tumors (NET) is challenging.

- Pathologic classification of thoracic NET should involve assessment of both proliferation rate (Ki-67 and mitoses) and cell differentiation (well, moderately or poorly differentiated).

- We describe a case of thoracic NET first misdiagnosed as high-grade neuroendocrine carcinoma, with no Ki-67 performed during classification, leading to the patient being unnecessarily treated with aggressive chemotherapy.

- Standard first-line treatment for high-grade neuroendocrine carcinoma consists of chemotherapy.

- Poor response to chemotherapies led to rediagnosis of this tumor as a typical carcinoid.

- Chemotherapy is not recommended for typical carcinoids unless there are no other feasible treatment options.

- Current treatment guidelines recommend everolimus as a first-line therapy for advanced and progressive thoracic carcinoids.

- This case highlights the importance of accurate initial diagnosis for optimal treatment selection.

Wereport the case of a patient initially diagnosed with a high-grade neuroendocrine carcinoma, which 5 years later was determined to have a low-grade typical carcinoid. The patient received radiotherapy and numerous chemotherapy regimens for treatment of a high-grade metastatic mixed large and small cell neuroendocrine carcinoma, without a significant response to any treatment. Subsequent imaging revealed widely metastatic disease and computed tomography-guided biopsy demonstrated a carcinoid tumor with no necrosis. The patient was started on temozolomide + capecitabine, long-acting octreotide and denosumab, with everolimus planned upon disease progression. Findings from this case study highlight the importance of accurate histopathologic classification of thoracic neuroendocrine tumors at diagnosis, to avoid the unnecessary administration of aggressive chemotherapy to patients with low-grade tumors.

First draft submitted: 2 June 2017; Accepted for publication: 20 September 2017; Published online: 26 October 2017

Keywords: carcinoid • diagnosis $\bullet$ neuroendocrine tumors $\bullet$ small cell carcinoma • treatment

Neuroendocrine tumors (NET) are a heterogeneous group of rare malignancies arising from neuroendocrine cells, with approximately $25 \%$ of NET originating in the respiratory tract [1]. Lung NET can be classified into four subtypes according to WHO criteria: typical carcinoids (TC); atypical carcinoids (AC); large cell neuroendocrine carcinoma (LCNEC) and small cell lung carcinoma (SCLC), based on histologic diagnostic criteria, including: cell size; cell morphology; mitotic index; architectural growth patterns and necrosis [2].

Two key characteristics that aid in distinguishing between lung NET subtypes and help guide treatment decisions are the degree of aggressiveness (mitotic rate and Ki-67 proliferation index) and the extent of necrosis [2]. TC and $\mathrm{AC}$ are well-differentiated, low- or intermediate-grade lung NET. TC are defined as tumors measuring $0.5 \mathrm{~cm}$ or

Future $\because \%$ Medicine 
more, with fewer than two mitoses per $2 \mathrm{~mm}^{2}$ of viable tumor area and lacking necrosis, while AC characteristically show 2-10 mitoses per $2 \mathrm{~mm}^{2}$, with focal necrosis [2]. LCNEC and SCLC are poorly differentiated, high-grade lung NET, both demonstrating a high mitotic rate of greater than ten per $2 \mathrm{~mm}^{2}$ and extensive necrosis [3]. LCNEC and SCLC are primarily distinguished from one another on the basis of cell size and other cytologic features [2]. While the utility of Ki-67 in distinguishing TC from AC is not yet established, it is a useful marker to distinguish carcinoids from LCNEC and SCLC, because the proliferation rate in these tumors is very high compared with carcinoids [2].

The WHO classification system for pulmonary NET subtypes are best applied to correctly handled and optimally fixed surgical specimens [3]. Subtle differences can make histopathologic classification difficult, particularly in small biopsies or fine needle aspiration cytology samples or due to sample handling/procedural errors [3,4]. In addition, NET cell proliferation rates can vary between different regions within the same tumor [4]. The mitotic rate and Ki-67 index may also be discordant; for example, the mitotic rate might indicate a low-grade tumor, while the Ki-67 index indicates a higher grade tumor [4]. Generally, NET grading is based upon the most aggressive (highly proliferative) tumor site or indicator.

Correct histologic classification of advanced lung NET at diagnosis is critical in guiding treatment decisionmaking. Standard first-line treatment for SCLC and LCNEC consists of chemotherapy, with cisplatin or carboplatin in combination with etoposide recommended for SCLC [1,5]. However, chemotherapy is not recommended for lung carcinoids unless there are no other feasible treatment options and may only be considered for advanced, aggressive AC (high-proliferating carcinoids) [6,7]. For advanced, progressive carcinoids, everolimus is recommended as first-line therapy, unless somatostatin analogues (octreotide long-acting repeatable [LAR] or lanreotide) may be considered (e.g., in TC with slow growth-expressing somatostatin receptors) [7]. Everolimus is the first agent to receive US regulatory approval for well-differentiated lung NET (TC and AC), based on the RADIANT-4 Phase III trial [8].

Diagnosis of LCNEC or SCLC as a lower-grade carcinoid tumor may constitute a missed opportunity for timely initiation of aggressive cytotoxic therapy. Conversely, diagnosis of a carcinoid tumor as LCNEC or SCLC may expose patients unnecessarily to chemotherapy toxicity, and place them at risk of early disease progression. While overinterpretation of carcinoids as a more aggressive tumor is a rare occurrence, this remains a very real risk in clinical practice [3].

Here, we describe the disease characteristics and management of a patient initially diagnosed with a high-grade neuroendocrine carcinoma, who was subsequently determined to have a low-grade TC.

\section{Case}

In November 2010, a 78-year-old Haitian male never-smoker presented with right arm pain. Radiographic imaging revealed a humeral mass. A proximal humerus biopsy revealed a high-grade, metastatic, mixed large and small cell neuroendocrine carcinoma, with no Ki-67 performed. The patient underwent surgery and had a rod placed to stabilize the bone; he received no adjuvant chemotherapy or radiation and was subsequently lost to follow-up.

The patient had no follow-up or surveillance imaging done for 2 years. In December 2012, the patient presented with pain and swelling in his shoulder region. Computed tomography (CT) imaging of the chest and right upper extremity revealed a large lytic lesion in the proximal right humerus, mediastinal mass and pulmonary nodules. In February 2013, chemotherapy with carboplatin + etoposide was initiated for six cycles along with palliative radiotherapy to the shoulder, followed by surveillance. Weekly paclitaxel was initiated in November 2013, followed by vinorelbine as monotherapy in November 2014; however, no significant response to either treatment was observed. In December 2014, a positron emission tomography/CT scan revealed widely metastatic disease. Treatment with carboplatin + etoposide was restarted in January 2015 and the patient completed six cycles of treatment by May 2015, with a best response of stable disease; he was then started on surveillance.

In January 2016, a chest CT revealed an increase in the large heterogeneous infiltrating mass involving the mediastinum. CT-guided biopsy of the mediastinal mass was performed in February 2016 and showed a carcinoid tumor with no necrosis, $<2$ mitoses per 10 high-powered fields and a Ki-67 proliferation index $<2 \%$. Immunohistochemistry was strongly positive for CgA and synaptophysin and negative for TTF-1, napsin A, prostate-specific antigen, prostate-specific antigen phosphatase and KX3 .

The patient was referred to our institution in March 2016. At this time, urine 5-hydroxyindoleacetic acid was elevated to $10.7 \mathrm{mg} / 24 \mathrm{~h}$ (reference range: $2-8 \mathrm{mg} / 24 \mathrm{~h}$ ) [9]. An Octreoscan showed a large infiltrative soft tissue mass in the mediastinum measuring $11.5 \times 11.5 \mathrm{~cm}$ with diffuse uptake, in addition to active lesions in the lung, 


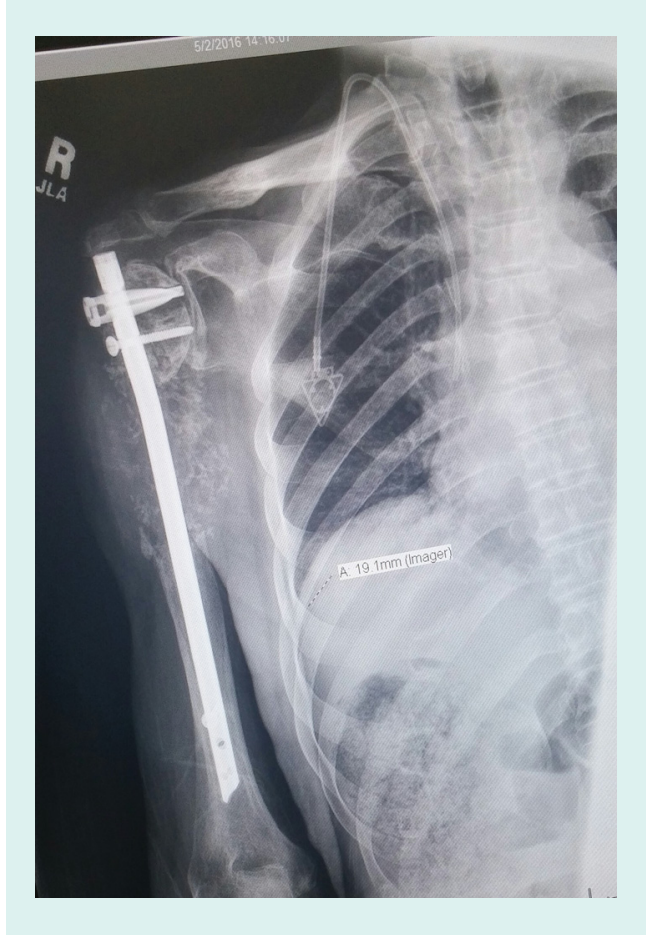

Figure 1. X-ray revealing a large, expansile and destructive lesion within the proximal right humerus and nodule densities in the lateral right lower lobe.

liver and bone. Based on the indolent natural course of disease, along with lack of significant response or progression throughout the course of several treatments, we considered the most likely diagnosis to be a low-grade carcinoid tumor (TC). The patient was started on treatment with temozolomide + capecitabine, along with octreotide LAR and denosumab.

In April 2016, x-ray imaging revealed a large, expansile and destructive lesion within the proximal right humerus (surgical hardware within the humerus appeared well seated and intact; Figure 1). Nodule densities in the lateral right lower lobe corresponded to pulmonary nodules, as observed in the chest CT scan from the previous month. The CT scan identified two adjacent hypermetabolic pulmonary nodules in the left lower lobe measuring $1.5 \times$ $1.2 \mathrm{~cm}$ with standard uptake value (SUV) 6.3 and $0.9 \times 1.1 \mathrm{~cm}$ with SUV 5.7. Additional pulmonary nodules were seen with lower level 18-fluorodeoxyglucose uptake, including a 0.5 -cm nodule in the superior segment of the right lower lobe, SUV 1.4 and a 0.9-cm nodule at the right lung base, SUV 2.2 (Figure 2). In August 2016, the patient presented with a mass on his right arm and palliative radiation therapy is planned (Figure 3 ). If the patient exhibits further evidence of disease progression, a switch to treatment with everolimus is planned.

\section{Discussion}

This case highlights the importance of accurate histopathologic classification of thoracic NET at diagnosis to ensure a timely and appropriate treatment approach. With this case, the eventual diagnosis of TC was only reached on the basis of the indolent disease course and lack of response to therapy. The initial diagnosis resulted in several iterations of suboptimal and aggressive systemic therapy, with a substantial delay ( $\approx 6$ years) in initiating appropriate treatment. While we rely on pathologists to make a histologic diagnosis, we must also include clinical judgment to guide our treatment decisions. The fact that the patient was a nonsmoker, as well as the indolent nature of the disease, were indicators that perhaps the initial biopsy was not providing us with the complete picture.

This case is similar to those of Pelosi et al. [3], who reported on a series of seven cases of lung carcinoids (two TC and five AC) which were initially diagnosed as SCLC. Crush artifact had occurred in five of the seven cases, which was likely to have contributed to the difficulties in tumor classification. As a result, six of the seven patients received preoperative cytotoxic chemotherapy before receiving a correct lung carcinoid diagnosis [3].

Such case reports underline the challenges faced by expert pathologists in definitively classifying thoracic NET samples. These difficulties were demonstrated in a study by den Bakkar et al. [10], who collected slides from 170 cases diagnosed as SCLC, LCNEC or general neuroendocrine lung carcinoma. The slides were distributed to 


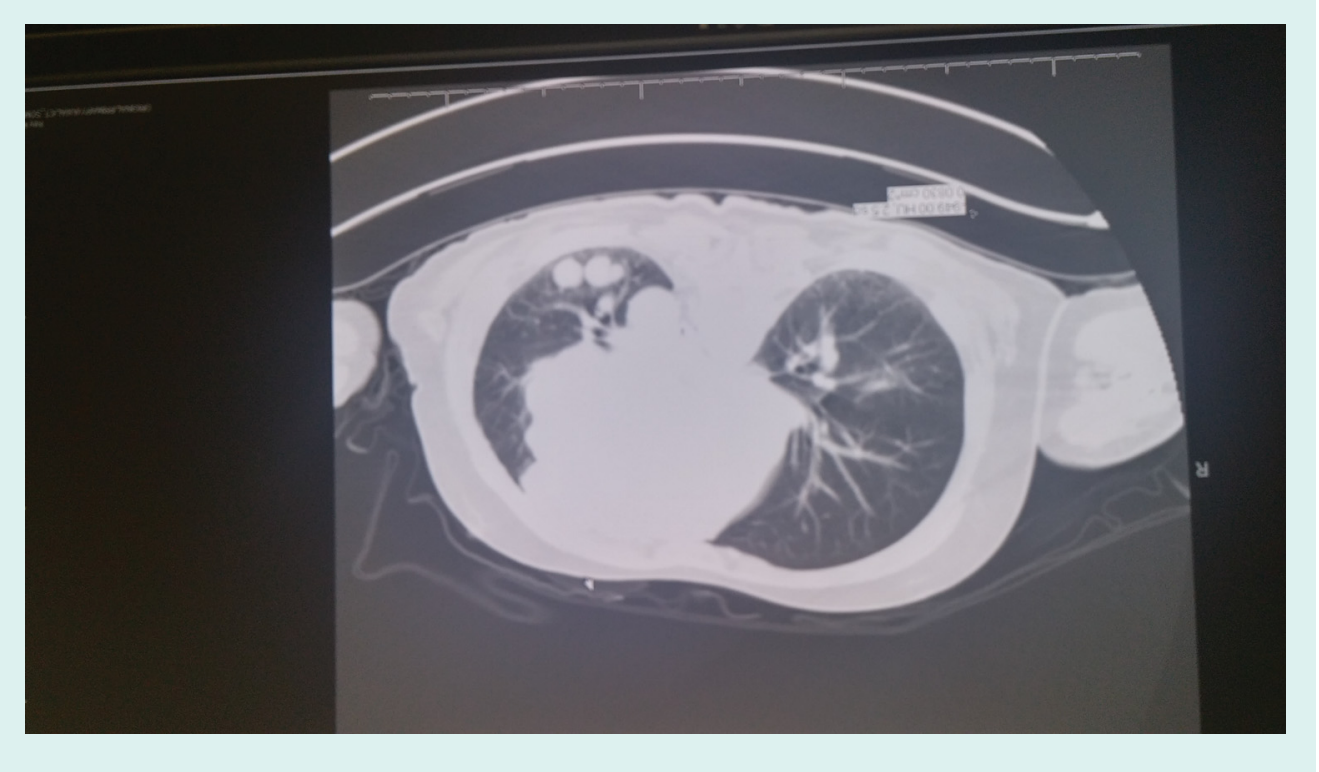

Figure 2. Recent computed tomography scan identifying two adjacent hypermetabolic pulmonary nodules in the left lower lobe.

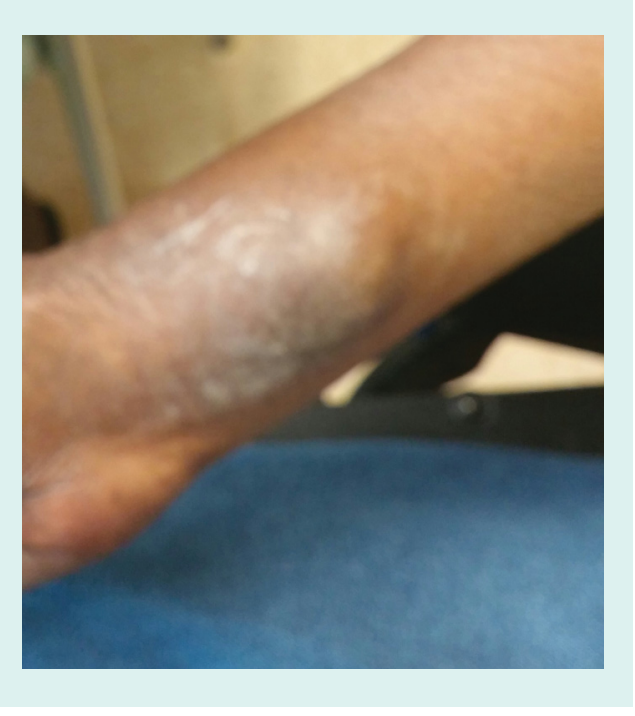

Figure 3. Mass on the patient's right arm in August 2016.

nine pathologists with a special interest in pulmonary pathology, who were asked to review and classify the samples using WHO criteria. There was a unanimous diagnosis made in only 20 cases, a majority consensus in 117 cases and no consensus in 33 cases. The number of cases diagnosed as SCLC varied from 49 to 109, while diagnoses of LCNEC varied between 14 and 85 cases [10].

Indicators of tumor aggressiveness are important markers for thoracic NET classification. In an analysis of 200 patients with pulmonary NET, Travis et al. [11] found that mitotic count was the only independent prognostic factor, underscoring the importance of accurate grading in this class of tumors. In addition, Ki-67 can help distinguish between carcinoids and other lung NET subtypes. Ki-67 immunoreactivity tends to be well preserved even in samples with crush artifact [3] and is considered to be the more reliable marker in cases where Ki-67 and the mitotic rate are discordant [4]. Also, in small cytologic samples, a greater number of cells are likely to express $\mathrm{Ki}-67$ than be in active mitosis [4]. The value of $\mathrm{Ki}-67$ as a marker was clearly demonstrated in the aforementioned 
article by Pelosi et al. [3]; compared with SCLC cases, the seven carcinoid cases had low Ki-67 indices (1-17\%), whereas all control cases of SCLC showed Ki-67 > 50\% (60-96\%).

With regard to other markers of interest, higher levels of $\mathrm{CgA}$ and synaptophysin immunoreactivity has been identified among carcinoid cases compared with SCLC [3]. TTF-1 is found infrequently in TC and AC; for example, only one of the seven cases in the report by Pelosi et al. showed focal immunoreactivity to TTF-1, but TTF-1 was consistently expressed among SCLC [3]. These markers, along with mitotic rate and Ki-67, should be considered together when classifying lung NET tissue samples.

\section{Conclusion}

From our clinical experience, we conclude that diagnosis of TC/AC as high-grade neuroendocrine carcinoma can lead to delays in the initiation of appropriate treatment or the use of ineffective, aggressive treatment approaches without clear benefit. Inappropriate treatment may also expose patients unnecessarily to excessive treatment-related toxicity and reduced quality of life. Improved awareness of the correct sample handling procedures and the nuances of known lung NET markers is expected to improve tumor classification accuracy and thus enhance patient outcomes.

\section{Acknowledgements}

We thank LM Balfe, PA-C, at the Sylvester Comprehensive Cancer Center and University of Miami Miller School of Medicine for assistance with preparation of the patient case materials.

Financial \& competing interests disclosure

M Jahanzeb has received research grants from Lilly, AbbVie, Genentech, Ipsen and Novartis. He has served as a consultant for AbbVie, Genentech, Ipsen and Novartis. The authors have no other relevant affiliations or financial involvement with any organization or entity with a financial interest in or financial conflict with the subject matter or materials discussed in the manuscript apart from those disclosed.

Medical writing assistance was provided by ApotheCom and was funded by Novartis Pharmaceuticals Corporation.

Informed consent disclosure

The authors state that they have obtained verbal and written informed consent from the patient/patients for the inclusion of their medical and treatment history within this case report.

\section{Open access}

This work is licensed under the Attribution-NonCommercial-NoDerivatives 4.0 Unported License. To view a copy of this license, visit http://creativecommons.org/licenses/by-nc-nd/4.0/

\section{References}

1 National Comprehensive Cancer Network. NCCN Clinical Practice Guidelines in Oncology. Small Cell Lung Cancer Version 2 (2017). www.nccn.org/professionals/physician_gls/pdf/sclc.pdf

2 Travis WD, Brambilla E, Burke AP, Marx A, Nicholson AG. WHO Classification of Tumours of the Lung, Pleura, Thymus and Heart. International Agency for Research on Cancer, Lyon, France (2015).

3 Pelosi G, Rodriguez J, Viale G, Rosai J. Typical and atypical pulmonary carcinoid tumor overdiagnosed as small cell carcinoma on biopsy specimens: a major pitfall in the management of lung cancer patients. Am. J. Surg. Pathol. 29(2), 179-187 (2005).

4 Klimstra DS. Pathology reporting of neuroendocrine tumors: essential elements for accurate diagnosis, classification, and staging. Semin. Oncol. 40(1), 23-36 (2013).

5 National Comprehensive Cancer Network. NCCN Clinical Practice Guidelines in Oncology. Non-Small Cell Lung Cancer Version 3 (2017). www.nccn.org/professionals/physician_gls/pdf/nscl.pdf

6 National Comprehensive Cancer Network. NCCN Clinical Practice Guidelines in Oncology. Neuroendocrine Tumors Version 2 (2016). www.nccn.org/professionals/physician_gls/pdf/neuroendocrine.pdf

7 Pavel M, O’Toole D, Costa F et al. All other Vienna Consensus Conference participants, ENETS Consensus Guidelines update for the management of distant metastatic disease of intestinal, pancreatic, bronchial neuroendocrine neoplasms (NEN) and NEN of unknown primary site. Neuroendocrinology 103(2), 172-185 (2016).

8 Yao JC, Fazio N, Singh S et al. For the RAD001 in Advanced Neuroendocrine Tumours, Fourth Trial (RADIANT-4) Study Group. Everolimus for the treatment of advanced, non-functional neuroendocrine tumours of the lung or gastrointestinal tract (RADIANT-4): a randomised, placebo-controlled, Phase III study. Lancet 387(10022), 968-977 (2016). 
9 Vinik A, Feliberti E, Perry RR. Carcinoid Tumors. [Updated 2014 Aug 1]. In: Endotext. De Groot LJ, Chrousos G, Dungan K et al. (Eds). MDText.com, Inc., MA, USA (2000). www.endotext.org/chapter/carcinoid-tumors/

10 den Bakker MA, Willemsen S, Grünberg K et al. Small cell carcinoma of the lung and large cell neuroendocrine carcinoma interobserver variability. Histopathology 56(3), 356-363 (2010).

11 Travis WD, Rush W, Flieder DB et al. Survival analysis of 200 pulmonary neuroendocrine tumors with clarification of criteria for atypical carcinoid and its separation from typical carcinoid. Am. J. Surg. Pathol. 22(8), 934-944 (1998). 